\title{
Análise da atividade da enzima creatina quinase e da isoenzima CK-MB - Simulação para ensino remoto
}

\section{Analysis of the activity of the enzyme creatine kinase and the isoenzyme CK-MB - Simulation for remote teaching}

Bruna Franchito Freire ${ }^{1 *}$, Paula Fernandes Gáspari², Celene Fernandes Bernardes ${ }^{3}$

${ }^{1}$ Institution: PUC-Campinas, ${ }^{2}$ Institution: PUC-Campinas, ${ }^{3}$ Institution: PUC-Campinas

*e-mail: brunafranchito@hotmail.com

\section{Resumo}

A análise da enzima creatina quinase $(\mathrm{CK})$ e da isoenzima CK-MB está prevista no plano de ensino da Disciplina de Bioquímica para o curso de Medicina. O intuito é ilustrar o tema de metabolismo energético anaeróbico e relacionar com casos clínicos de alterações do tecido cardíaco e infarto agudo do miocárdio. Com o uso de ferramentas acessíveis, como os programas Paint e PowerPoint, o tema foi desenvolvido de forma remota, utilizando simulações para demonstrar as etapas dos experimentos. O material foi apresentado aos alunos e disponibilizado na forma de vídeo. A apresentação e discussão do tema no formato virtual foi bem aceita pelos alunos, embora tenham relatado a importância das aulas presenciais para a formação médica.

Palavras-chave: creatina quinase; aula remota; Bioquímica experimental

\begin{abstract}
The analysis of the enzyme creatine kinase (CK) and the isoenzyme CK-MB is foreseen in the teaching plan of the Biochemistry discipline for the course of Medicine. The goal is to illustrate the topic of anaerobic energy metabolism and relate it to clinical cases of cardiac tissue changes and acute myocardial infarction. Using accessible tools, such as the Paint and PowerPoint softwares, the theme was developed remotely, using simulations to demonstrate the stages of the experiments. The material was presented to students and made available as a video. The presentation and discussion of the topic in the virtual format was well accepted by the students, although they reported the importance of face-to-face classes for medical training.
\end{abstract}

Keywords: creatine kinase; remote class; Experimental biochemistry 
Ficha da aula

\begin{tabular}{|c|c|}
\hline Título & $\begin{array}{l}\text { Análise da atividade da enzima creatina quinase e da isoenzima CK-MB - } \\
\text { Simulação para ensino remoto }\end{array}$ \\
\hline Tipo & Prática ( x ) \\
\hline Público-alvo & Alunos de Cursos de Medicina \\
\hline $\begin{array}{l}\text { Disciplinas } \\
\text { correlatas }\end{array}$ & Bioquímica Geral e Bioquímica aplicada à Área Médica \\
\hline $\begin{array}{l}\text { Objetivos } \\
\text { educacionais }\end{array}$ & Estudo da atividade da enzima creatina quinase e da isoenzima CK-MB \\
\hline Justificativa & $\begin{array}{l}\text { O estudo do tema possibilitará: } \\
\text { - Ilustrar e aprofundar conhecimentos teóricos relacionados ao metabolismo } \\
\text { energético anaeróbico e alático; } \\
\text { - Identificar os marcadores bioquímicos plasmáticos para diagnosticar e } \\
\text { acompanhar o tratamento de alterações cardíacas, em especial do infarto } \\
\text { agudo do miocárdio; } \\
\text { - Relacionar os resultados das análises com casos clínicos; } \\
\text { - Compreender como os exames clínicos bioquímicos são realizados; } \\
\text { - Desenvolver a percepção das possibilidades de ocorrência de erros técnicos } \\
\text { que poderão ocasionar falsos resultados nos exames; } \\
\text { - Desenvolver habilidades no manuseio dos instrumentos utilizados em } \\
\text { laboratório de bioquímica. }\end{array}$ \\
\hline $\begin{array}{l}\text { Conteúdos } \\
\text { trabalhados }\end{array}$ & $\begin{array}{l}\text { Metabolismo anaeróbico muscular } \\
\text { Alterações celulares em hipóxia } \\
\text { Marcadores plasmáticos do Infarto Agudo do Miocárdio }\end{array}$ \\
\hline Duração & 3 horas/aula \\
\hline $\begin{array}{l}\text { Materiais } \\
\text { usados }\end{array}$ & $\begin{array}{l}\text { Programa de computador Paint e PowerPoint para a aula na forma remota. } \\
\text { Materiais necessários para a aula presencial: } \\
\text { Padrão da enzima creatina quinase para a análise controle; } \\
\text { Amostra de soro ou plasma sanguíneo; } \\
\text { Reativos específicos para análise enzimática colorimétrica de CK e CK-MB; } \\
\text { Tubos de ensaio, pipetas, banho-maria a } 37^{\circ} \mathrm{C} \text {, espectrofotômetro para leitura } \\
\text { de absorbância na faixa de } 365 \mathrm{~nm} \text {. }\end{array}$ \\
\hline Links & https://youtu.be/ZN8LXs2217M \\
\hline
\end{tabular}




\section{Introdução}

O ensino de Bioquímica nos cursos de Medicina é muito importante para preparar os futuros médicos para situações que encontrarão em suas carreiras. A análise dos componentes celulares é uma grande aliada para o diagnóstico de diversas doenças.

As aulas práticas de Bioquímica são normalmente realizadas em laboratórios específicos e desenvolvidas com temas previamente estudados na teoria. No entanto, em 2020, em meio ao isolamento social, foi necessário o uso de novas ferramentas para dar continuidade ao ensino prático da disciplina. Em relato anterior mostramos que com o uso de métodos simples, com ferramentas acessíveis como os programas Paint e PowerPoint, foi possível realizar aula prática com simulação esquemática de experimentos para 0 ensino das moléculas de carboidratos. A temática anterior é extremamente genérica na Bioquímica e ministrada em diferentes cursos; no entanto, as aulas práticas simuladas também possibilitaram o ensino de processos relacionados com a Medicina. Assim, foram realizadas simulações online para o estudo experimental da atividade da enzima creatina quinase (CK, EC 2.7.3.2) e da isoenzima CK-MB a fim de exemplificar e contextualizar a Bioquímica com casos clínicos.

A CK participa na homeostase energética das células. A molécula é constituída de dois dímeros ( $\mathrm{M}$ e $\mathrm{B}$ ), formando três isoenzimas citosólicas, duas homodímeras (CK-BB e CK-MM) e uma heterodímera (CK-MB) [1-6]. Essas apresentam diferentes atividades nos tecidos e caracterizam alterações celulares específicas [3-6]. Lesão no tecido cerebral é evidenciada pelo aumento de CK-BB plasmática; enquanto, em lesão no músculo cardíaco, a isoenzima prevalente é a CK-MB [3-6].

Nas aulas de Bioquímica para o curso de Medicina, a análise experimental da CK e da CK-MB foi muito importante para que, mesmo durante o isolamento social, os alunos pudessem compreender como os resultados laboratoriais são obtidos e a relação com parâmetros que possibilitam o diagnóstico e o acompanhamento do infarto do miocárdio [7-11].

Os alunos relataram que a experiência foi muito enriquecedora. Além disso, embora acreditem que as aulas presenciais sejam de grande importância e indispensável para a formação médica, concluíram que o uso dessas ferramentas online foi essencial para dar continuidade ao curso. 


\section{Análise da atividade da enzima CK e da isoenzima CK-MB}

\subsection{Fundamentos teóricos}

A atividade da CK e da CK-MB foi verificada analisando o aumento de absorbância decorrente do NADPH, formado por meio das reações demonstradas abaixo [3,12]. O acréscimo de um anticorpo inibidor da subunidade $\mathrm{M}$ permite analisar apenas a atividade do monômero CK-B e relacionar com a atividade da CK-MB $[3,13]$.

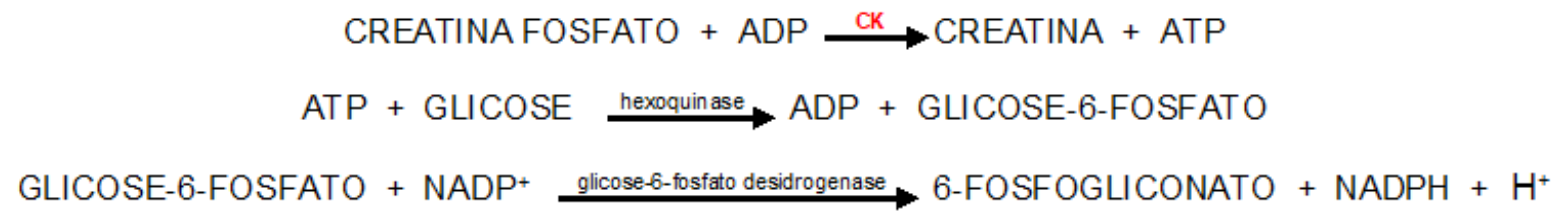

O processo foi realizado, de forma comparativa, na amostra de soro do paciente e numa enzima padrão, com atividade enzimática previamente estabelecida.

\subsection{Procedimentos experimentais e resultados}

A atividade da CK [12] e da CK-MB [13] foram determinadas conforme esquemas representados na figura 1.
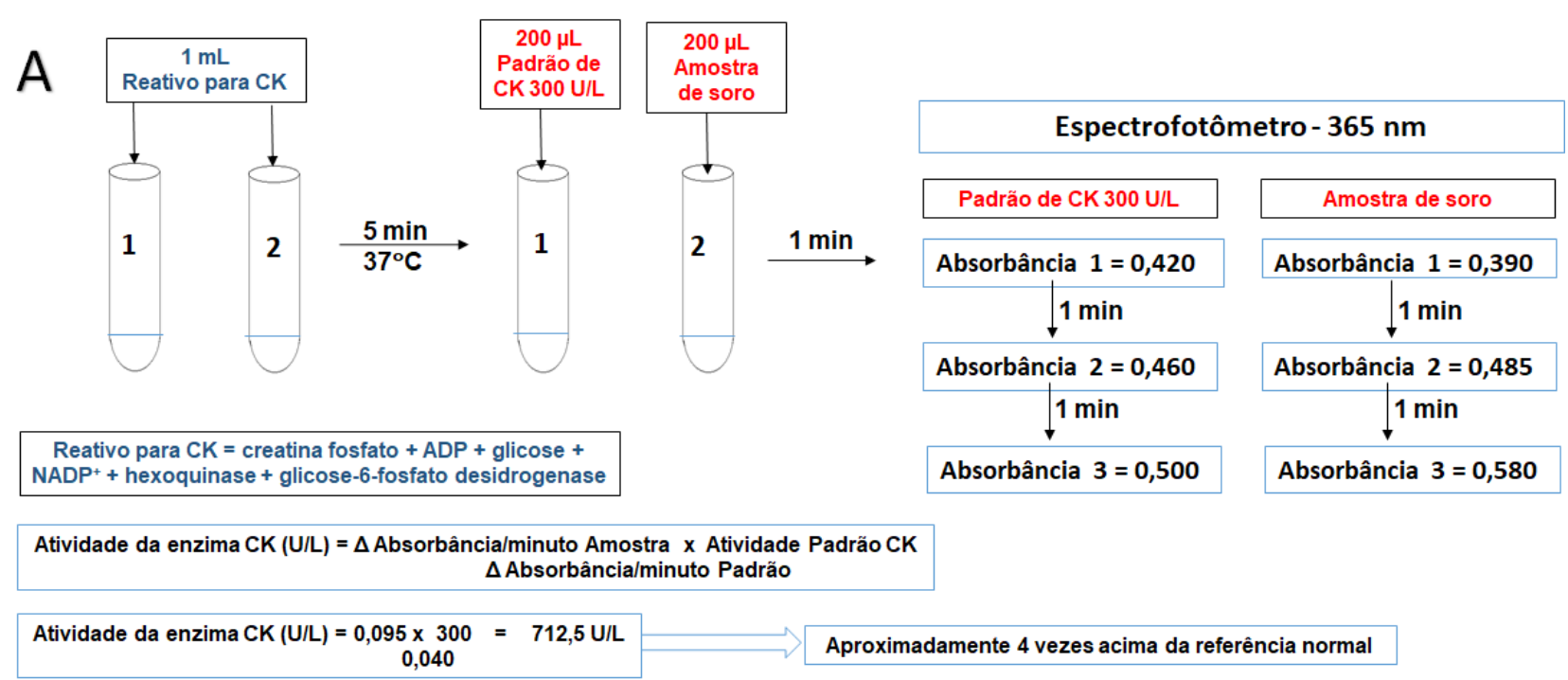

Referência = homens até $171 \mathrm{U} / \mathrm{L}$ e mulheres até $145 \mathrm{U} / \mathrm{L}$ 


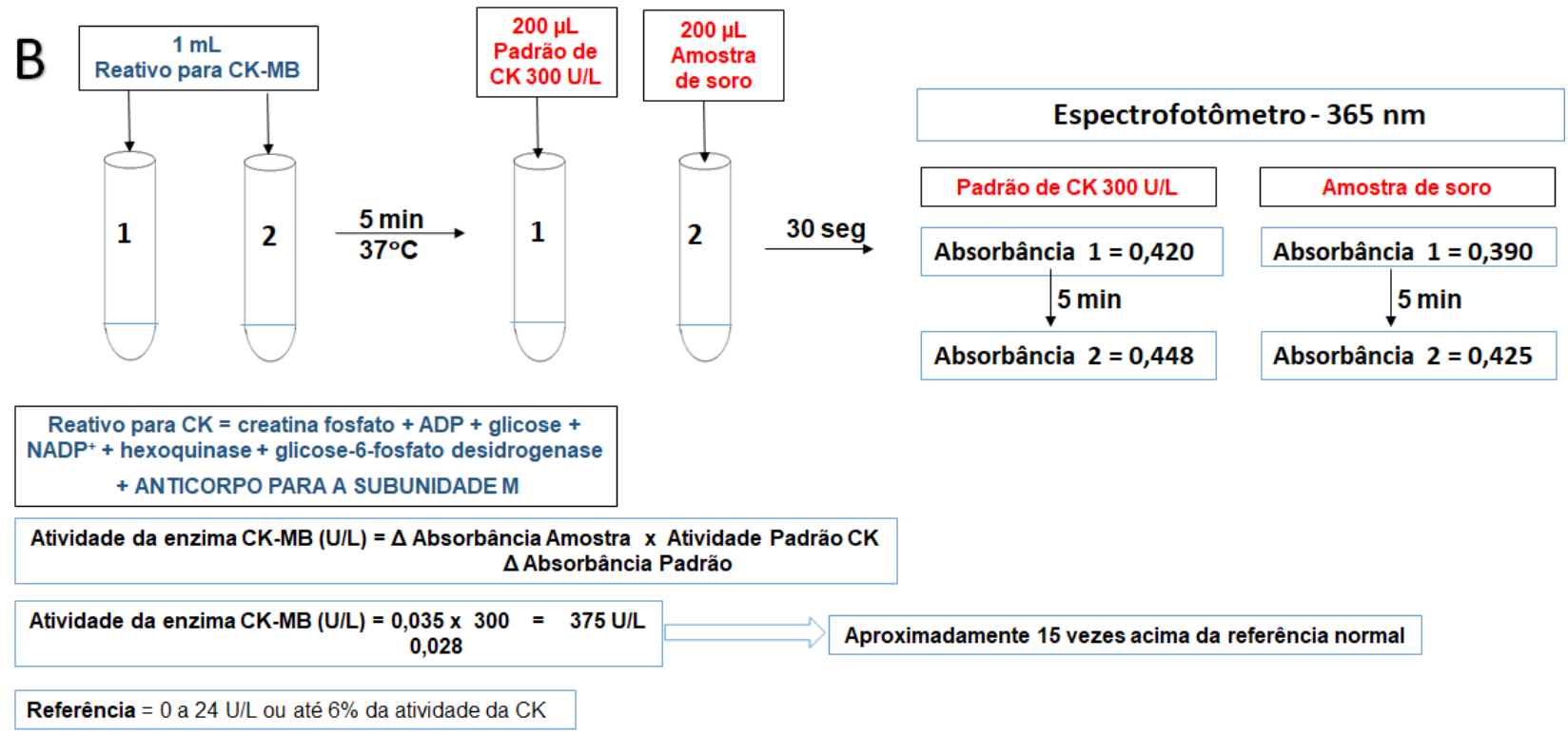

Figura 1 - Análise da atividade da enzima CK (A) e da isoenzima CK-MB (B)

\section{Interpretação dos resultados:}

Os resultados, interpretados conforme Figura 2, indicaram que a amostra de soro tem grande probabilidade de ser originária de um indivíduo com diagnóstico de Infarto agudo do miocárdio (IAM).

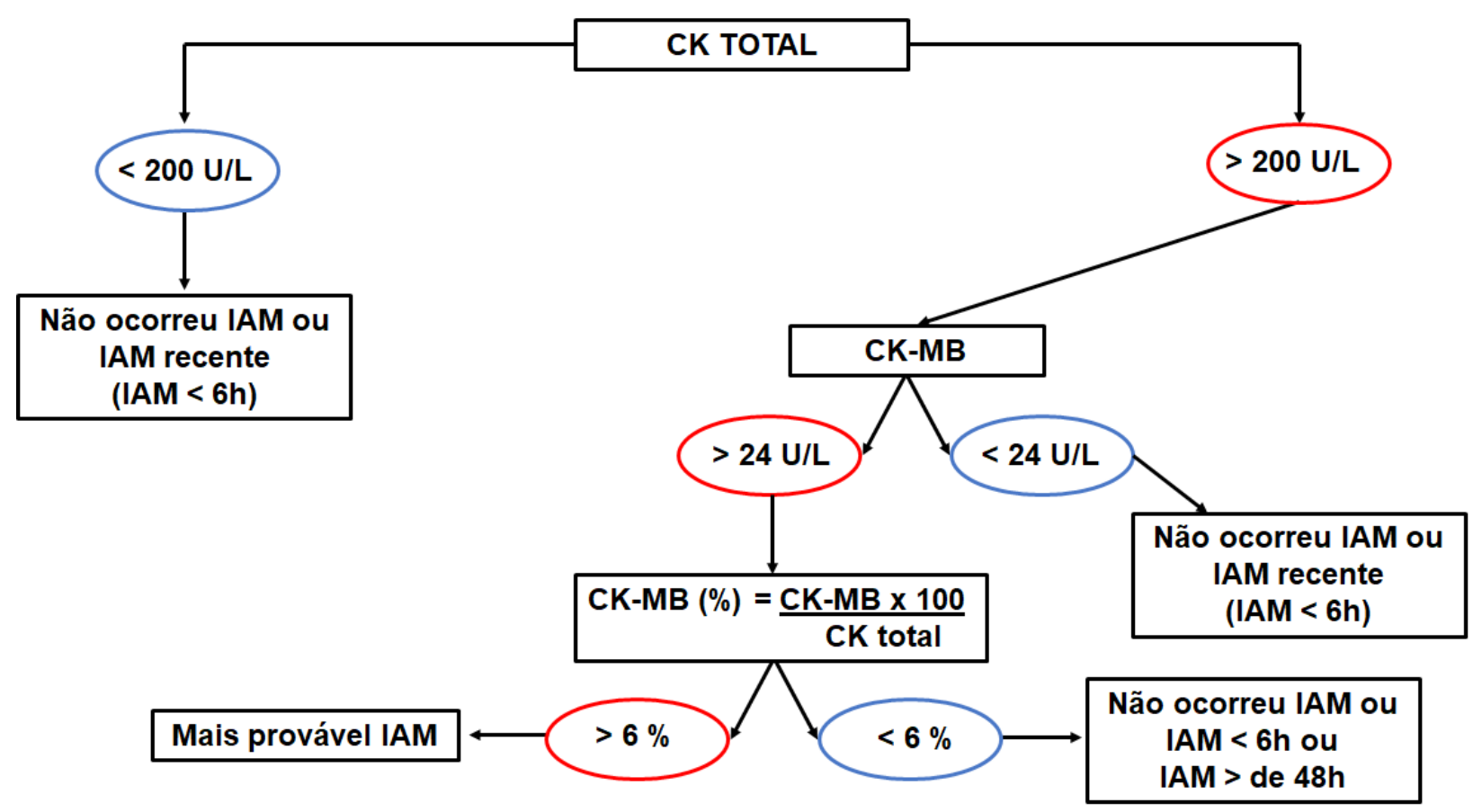

Figura 2 - Interpretação dos resultados para avaliação do IAM

Fonte: Adaptado de CK-MB Liqueform [13] 


\section{Referências}

[1] Bong S. M. et al. Structural studies of human brain-type creatine kinase complexed with the ADP-Mg2+NO3- -creatine transition-state analogue complex. FEBS Lett 582, 3959-3965 (2008).

[2] George R, Wallage M, Goodall R. The use of trypsin to confirm the presence of macrocreatine kinase on isoenzyme electrophoresis. Ann Clin Biochem. 2012;49(Pt 4):359-62.

[3] Burtis CA, Ashwood ER, Bruns DE. Fundamentos de Química Clínica. 6. ed. Rio de Janeiro: Elsevier, 2008.

[4] Wang Y, Wang S, Gao YS, Chen Z, Zhou HM, Yan YB. Dissimilarity in the folding of human cytosolic creatine kinase isoenzymes. PLoS One. 2011;6(9):e24681.

[5] Marshall WJ, Lapsley M, Day AP, Ayling RM. Bioquímica Cínica - aspectos clínicos e metabólicos. 3. ed. Rio de Janeiro: Elsevier, 2016.

[6] Schlattner, U., Klaus, A., Ramirez Rios, S. et al. Cellular compartmentation of energy metabolism: creatine kinase microcompartments and recruitment of B-type creatine kinase to specific subcellular sites. Amino Acids 48, 1751-1774 (2016).

[7] Huerta-Alardín AL, Varon J, Marik PE. Bench-to-bedside review: Rhabdomyolysis -- an overview for clinicians. Crit Care. 2005 Apr;9(2):158-69.

[8] Jacob R, Khan M. Cardiac Biomarkers: What Is and What Can Be. Indian J Cardiovasc Dis Women WINCARS. 2018;3(4):240-244.

[9] Ye XD, He Y, Wang S, Wong GT, Irwin MG, Xia Z. Heart-type fatty acid binding protein (H-FABP) as a biomarker for acute myocardial injury and long-term post-ischemic prognosis. Acta Pharmacol Sin. 2018 Jul;39(7):1155-1163.

[10] Aydin S, Ugur K, Aydin S, Sahin I, Yardim M. Biomarkers in acute myocardial infarction: current perspectives. Vasc Health Risk Manag. 2019 Jan 17;15:1-10.

[11] Bostan MM, Stătescu C, Anghel L, Șerban IL, Cojocaru E, Sascău R. Post-Myocardial Infarction Ventricular Remodeling Biomarkers-The Key Link between Pathophysiology and Clinic. Biomolecules. 2020 Nov 23;10(11):1587.

[12] CK-NAC Liquiform. Vista Alegre, MG: Labtest Diagnóstica S.A.; 2014.

[13] CK-MB Liquiform. Vista Alegre, MG: Labtest Diagnóstica S.A.; 2011. 